\title{
Importancia
}

\author{
Ernesto lópez Bonilla', Lucio Rojas Cortés ${ }^{2}$ \\ ana Rosa Torres Moreno ${ }^{3}$
}

\section{Estratégica del}

\section{Cuadro Integral}

de Mando en

la Gestión

\section{Empresarial}

Balanced

\section{Scorecard}

\section{RESUMEN}

$\mathrm{E}$ I modelo de Balanced Scorecard o Tablero integral de Mando, es una poderosa herramienta de planeación estratégica que permite a la empresa moderna crear y controlar sus propios indicadores de gestión relacionados dentro de cuarro perspectivas, a saber: Financiera, Procesos Incernos, Cliente y Formación y Crecimiento, encrelazándolos con la misión. la visión y contemplando los aspectos internos externos que puedan afectar la cadena de valor de la organización.

\section{INTRODUCCIÓN}

Frente a un mercado globalizado, diná. mico, altamente competitivo y con grandes dosis de incertidumbre, la empresa moderna

1 Connador Público, Universidad de fa Salle. Especialización Gerencia Estratégica de Costos. Docente: Fundación Univasiojid Centraf, Universidad INCA de Colombia, UniversidaA Libre.

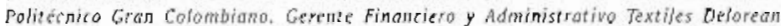
2 Lic. en Matemáticas: Universidad Distrital. Especializoción Gerentcia Estratégica de Costos Docenie: Fundacion Universidad Central. Universidad Militar. Pontificia Universidad Javeriana 3 Feonomisto. Fundocion Uxiverşidad de Bogota Jorge Tadeo Lozono. Estadistico Admiinstrativo: Fundación Unjversidad Jorge Tadeo Lozano. Especialización en Gerencia Estrategica de Costos. Docenze: Fundación Universidad de Bogotá forge Tadeo Lozano, Funúcion Liniversidad Central 
busca, cada vez más, contar con elementos que le faciliten disminuir la distancia que separa las compañías económicas y tecnológicas más avanzadas, de aquellas que no lo son.

La dirección empresarial requiere contar con una serie de análisis siscemáticos de sus fortalezas y debilidades, no sólo en el nivel interno, sino a un nivel de factores que aparentemente no tienen incidencia directa sobre sus actividades. Entre estos aspectos podemos considerar todos los factores del entorno externo a la empresa, cuya acción finalmente afecta los resultados económicos.

Lo anterior, se auna para crear la necesidad de adoprar técnicas y elementos que permitan llevar a cabo uma adecuada toma de decisiones. Tales elementos se encuentran concentrados en informes y dacos que son parte vital de la llamada información para la Dirección o Gestión Enpresarial.

El presente documento pretende presentar una alternativa sólida de Gestión Estratégica, que conduce a las organizaciones a mejorar las dinámicas de las actividades y se constituye en un soporte para el control y desempeño de la función gerencial, llamada Balanced Scorecard - Cuadro de Mando Integral; en él se cntrelazan todas las actividades que competen a la empresa (Internas - Externas) encajadas dentro de cuatro perspecivas: Financiera, Recursos Internos, Cliente e Investigación y Desarrollo. mediante indicadores eslabonados y cruzados por la relación causa efecto; balanceados a corto y largo plazo y ligados a la misión y visión de la organización.

\section{RESEÑA HISTÓRICA:}

En las últimas décadas del siglo $X X$ el manejo de grandes flujos de información trajo consigo muchos cambios en los procedimiencos de planeación y de control estratégico, pero esto no significa que durante todo el siglo los estudiosos del tema no hayan hecho propues- tas con respecto a la materia. La preocupación generada por la insuficiencia práctica de indicadores de gestión hacia 1910-1912 originaron estrategias basadas en la implementación de indicadores por áreas claves para el desarrollo de la empresa, destacándose entre ellos LOS INDICES DE DUPONT, instrumento, que aún hoy se encuentra vigente.

Para la década de 1950, Peter Drucker, como asesor de la General Electric, implanta una serie de Indicadores de Control para las áreas neurálgicas de la Empresa, los que han hecho carrera y se han posicionado como instrumentos de evaluación en nuchas empresas modernas.

En la misma década, la Universidad de Harvard (1953), dada su preocupación por el manejo del control presupuestal y las dificultades administrativas que ello conlleva, emite un articulo sobre el tema.

Hacia el año 1968, las inquietudes sobre la materia continúan planceadas y surgen entonces, Métodos de Administración de los Sis. temas de Información, y se generan perspectivas para su manejo. Hasta entonces la aplicación de los procedinientos de gesción se desarrollaba dentro de un mundo estable con administraciones jerárquicas, centralizadas y de baja comperencia.

La globalización de las economías y el desarrollo tecnológico trajeron, en los años setenta, empresas más competitivas y dinánicas. Se hace necesario entonces, satisfacer al cliente al mismo tiempo que se requiere un mayor control en los costos de fabricación; este sistema exige un modelo más dinámico de proyección y planeación. Se requieren administraciones menos acartonadas, que dispongan de medios de control más ágiles y efectivos, de visión horizontal, que permitan modificaciones a futuro, conlleven acciones concretas, se basen en indicadores físicos, con planes de acción, 
previsión e información operativa integrada y coherente.

La Universidad de Harvard se interesa en el tema y lo demuestra a cravés de publicaciones realizadas por sus investigadores David Norton y Robert Kaplan, en una serie de artículos publicados en la revista Harvard Business Review como The Role for Empirical Research in Management Accounting (1994) por Kaplan R.S., The Balanced Scorecard - Mesures that Drive Performance (1992) por Kaplan R. S., y Norton D., Putting the Balanced Scorecard to Work (1993) por Kaplan R. S. Y Norton D. Using Scorecard the Balanced as a Strategic Management Sistem (1996) por Kaplan R. S. Y Norton D. Estos artículos dieron origen en 1996 a un texto que se ocupa de manera directa del control de gestión, "The Balanced Scorecard: translating strategy into action"; en ésce los aultores, Robert S. Kaplan y David Norcon, plancean inquietudes frente a la opción de manejar un sistema unificado y balanceado de información gerencial que hoy se conoce como Tablero de Control de Balance, Tablero Integral de Control, o Tablero de Mando Integral.

La técnica se nutre de los conceptos generados en las teorías modernas de gestión organizacional (Gerencia de la Calidad Total, Procesos de Reingeniería, Costeo Basado en Actividades y Valor Económico Agregado (EVA)) entre otros, manejados integralmente y orientados a la creación de valor para la empresa, para el cliente y para el entorno; todo encajado en la relación causa efecto y enlazando la misión y la visión dentro de cuatro perspectivas, a saber: Financiera, Recursos Internos, Cliente e Investigación y Desarrollo.

\section{EL BALANCED SCORECARD \\ (Tablero de Mando Integral)}

\section{ASPECTOS GENERALES}

La propuesra de este novedoso sistema, como ya se enunció, es integrar las diferentes teorías de administración dentro de cuatro perspectivas: Financiera, Recursos Internos, Cliente e Investigación y Desarrollo, mediante indica. dores eslabonados y cruzados por la relación causa efecto; balanceados a corto y largo plazo, financieros y no financieros, internos y externos, históricos y proyeciados, de resultado y de proceso, a fin de garantizar que la toma de decisiones se lleve a cabo dentro de un ambiente de menor incertidumbre.

El Tablero Integral de Mando se constituye, pues, en la actualidad. en una herramienta invaluable para el manejo de la información relacionada con la toma de decisiones a nivel gerencial, ya que permite a cada empresa, a cada directivo y a cada responsable por área, diseñar, crear, controlar, manejar e interpretar sus propios indicadores, no sólo en el nivel interno de la organización, sino también en aspectos externos, que en muchos casos, aparentemente tienen poca o ninguna relación con el desempeño de las empresas, pero que al observarse con detenimiento señalan claras implicaciones en el rendimiento interno.

El cuadro ideado por Norton y Kaplan (pág. 20) que se ve a continuación, resume la técnica y señala la estructura para transformar la estrategia en términos operativos.

Cuando nos encontramos en un mercado altamente competido, con clientes y competidores mejor informados, con una mayor intervención del estado y de los gremios en el desempeño empresarial, con un amplio y cada vez más ágil manejo de los sistemas de comunicación, no es suficiente la utilización de indicadores financieros y económicos. Se requiere observar con detenimiento aspectos tales como la productividad, satisfacción de clientes y proveedores, compromiso del personal de la organización, aspectos logísticos, costos por desarrollo de accividades, calidad de la manufaccura y el servicio, ciclo de vida de las empresas y productos, agilidad en los procesos, capacidad de res- 


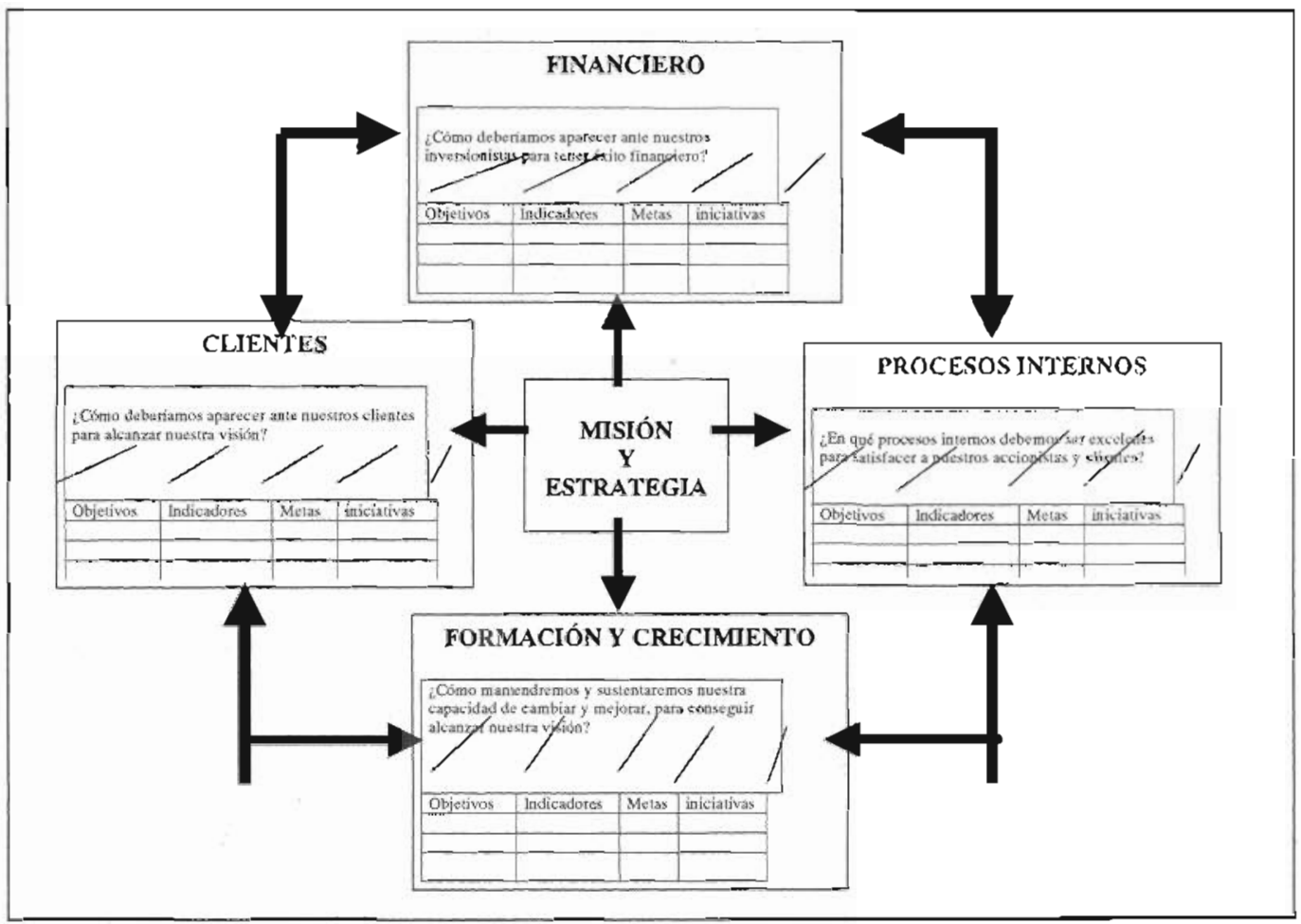

puesta, nivel de competitividad, liderazgo, medición de rentabilidad, productividad, capital intelectual, entre otros, que deben quedar claramente establecidos en los formatos de control de los objetivos, los indicadores, las metas $y$ las iniciativas.

El B.S.C. busca actuar como elemento integrador de los hechos actuales con los futuros, de tal manera que la atención gerencial esté puesta en el horizonte, apoyada en el permanente intercambio de datos, experiencias y opiniones con los involucrados directa o indirectamente en el proceso mediante la relación causa - efecto, expresada ésta, como un silogismo de tipo: Si...l Entonces.... estableciendo un vínculo entre la mejora de los objetivos propuescos y los beneficios obtenidos. El sistema de indicadores debe hacer que las relaciones (Hipótesis) entre los objetivos e indicadores en las diversas perspectivas sean explícitas a fin de ser gestionadas y rerroalimentadas.

\section{EL BALANCED SCORECARD DESDE LAS CUATRO PERSPECTIVAS}

\section{Financiera}

En la perspectiva financiera el B.S.C., retiene los indicadores financieros, como mediciones de hechos históricos y como elementos de control de la ejecución, Esta perspecriva se enfoca con énfasis en los resultados de rentabilidad y generación de valor para la empresa.

En ésta el B.S.C. vincula las otras perspectivas, pero cuidando de no incluir los mismos indicadores de rendimiento para todas las áreas de la organización o para las diferentes fases del 
ciclo de vida de la empresa (crecimiento, sostenimiento y cosecha).

De manera, pues, que la responsabilidad del área financiera tiene que cambiar de un ente controlador, como el que ha venido desempeñando, a otro con características de impulsor, esto es dejar de mirar al pasado para enfocarse hacia el futuro.

Los objetivos financieros deben ser revisados periódicamente, bien sea, para reafirmarlos o para cambiarlos.

\section{De procesos operativos internos}

Para enfrentar los problemas que afronta esta dirección tanto en lo relacionado con la obtención óptima de la mezcla de productos terminados como con las tecnologías en procesos productivos a emplear, se requiere administrar información pertinente a las cres funciones básicas de producción que aparecen en la mayoría de las organizaciones.

- Planeación y control de la producción.

- Dirección de la fabricación.

- Dirección de servicios.

E) B.S.C., en el área de procesos intermos está atento en el control y mejora de los Centros de Responsabilidad existentes. Los indica- dores se fortalecen con la aplicación de aspectos como la calidad, rendimiento. producción y tiempos de ciclo.

El Cuadro de Mando Integral tiene como objetivo La Excelencia; para lograrlo, se debe tener en cuenta el modelo de Cadena Cerencial de valor.

En el área de producción se enfatiza en el control de procesos y la estructura de costos. Siempre manteniendo el pumto de equilibrio (costos fijos / margen de contribución) y buscando un apalancamiento operativo adecuado.

\section{Del cliente}

Es conveniente identificar el segmento de población al cual se van a dirigir los esfuerzos de mercadeo, tanco de clientes como de empresas seleccionadas, cuota retención, incremento, satisfacción y rencabilidad, estos serán los objetivos para el desarrollo de procesos de Marketing, operaciones, logistica, productos y servicios que ofrecen satisfacción al cliente (interno y externo), con servicios o productos que terminen siendo valorados por ellos. Los objetivos deben ser concretos aunque cada organización debe establecer los propios, bajo el principio de que el interés del cliente está encaminado al tiempo de respuesta, la calidad, el precio y la relación entre proveedor y consumidor.

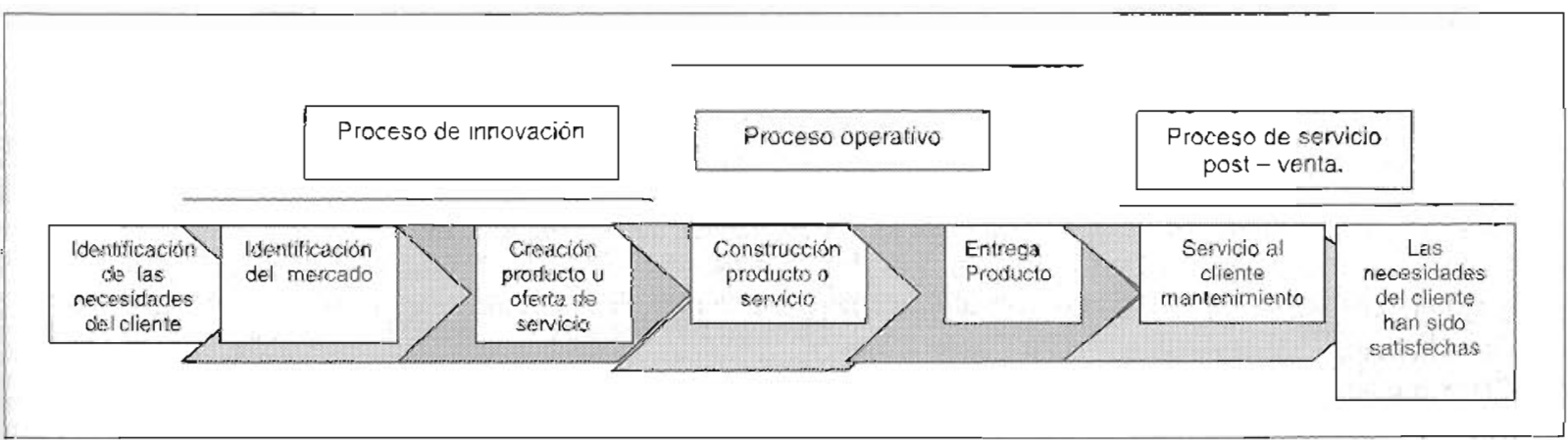

IFuente "Cuadro de Mando Inlegral" Kaplan Roberts y Norton Dovid P. Pag 1121 


\section{Aprendizaje y crecimiento}

Bajo el diseño del B.S.C., los negocios tendrán que invertir en la actualización de empleados, potenciar los sistemas y la tecnología de la información y coordinar los procedimientos y rutinas de la organización.

Los inductores de creciniento y aprendizaje provienen de los empleados, los sistemas y la equiparación de la organización; estos indicadores deben esrar basados en los empleados, su satisfacción. produccividad y retención.

El director de recursos humanos ha de establecer las estracegias, políticas y planes de la empresa en maceria de personal, que permi- tan la capacicación y motivación de recursos humanos en la relación con los valores, desarrollo y objetivos de la empresa.

\section{IMPLEMENTACIÓN DEL BALANCED SCORECARD}

Para la implantación de un modelo de Balanced Scorecard se debe comenzar fijando los centros claves de éxito para la empresa, y lue go proponer las variables y los indicadores que permitan conseguir los objerivos marcados.

López Viñegla, en el Cuadro de Mando y los Siscemas de Información para la Gestión Empresarial (pág. 154), propone la siguiente estructura para organizar los factores claves de la gestión empresarial.

\begin{tabular}{|c|c|}
\hline PRODUCCIÓN & $\begin{array}{l}\text { CALIDAD } \\
\text { NIVEL DE PRODUCCIÓN } \\
\text { INVENTARIOS } \\
\text { COSTOS LIDERAZGO } \\
\text { TECNOLÓGICO }\end{array}$ \\
\hline PRODUCTIVIDAD & $\begin{array}{l}\text { PRODUCTIVIDAD GLOBAL } \\
\text { PRODUCTIVIDAD DE LA EMPRESA } \\
\text { PRODUCTIVIDAD DEL TRABAJO }\end{array}$ \\
\hline CLJENTE & $\begin{array}{l}\text { SATISFACCIÓN DEL CLIENTE } \\
\text { CALIDAD DEL PRODUCTO }\end{array}$ \\
\hline RECURSO HUMANO & $\begin{array}{l}\text { COMPOSICIÓN DE LA NOMINA } \\
\text { NIVEL SALARIAL } \\
\text { AMBIENTE DE TRABAJO } \\
\text { SEGURIDAD E HIGIENE } \\
\text { FORMACIÓN Y PROMOCION }\end{array}$ \\
\hline SITUACIÓN FINANCIERA & $\begin{array}{l}\text { RENTABILIDAD ECONOMICA Y FINANCIERA } \\
\text { MARGENES DE ROTACIÓN } \\
\text { ESTRUCTRURA FINANCIERA } \\
\text { INVERSION Y PLAZOS DE FINANCIACION } \\
\text { COSTOS FINANCIEROS }\end{array}$ \\
\hline SITUACIÓN COMERCIAL & $\begin{array}{l}\text { VENTAS Y CUOTAS DE MERCADO } \\
\text { DEMANDA GLOBAL } \\
\text { DISTRIBUCION Y PUBLICIDAD } \\
\text { COMPRAS } \\
\text { COMPETENCIA } \\
\text { NUEVOS PRODUCTOS }\end{array}$ \\
\hline TECNOLOCÍA & $\begin{array}{l}\text { SISTEMAS DE ANÁLISIS DE INFORMACION } \\
\text { SITEMAS DE DISTRIBUCIÓN DE INFORMACIONN } \\
\text { INVESTIGACIÓNY DESARROLLO }\end{array}$ \\
\hline
\end{tabular}


Según experiencias en empresas colombianas, una de las posibles alternativas para la construcción de un B.S.C. está en contar la historia de la unidad de negocio, generar la Matriz DOFA 4 , de la empresa, proponer un objetivo estratégico específico y señalar las estrategias que estén direccionadas con las metas propuestas en la misión, la visión y los demás objetivos estrarégicos, establecer los indicadores de gestión para cada área en particular y entrelazarlos dentro de las cuatro perspectivas cuidando la relación causa efecto.

A manera de ejercicio se presentan a continuación algunos pasos que pueden tenerse en cuenta para la implementación (B.S.C).

1. Traducir cada estrategia en objetivos e indicadores, dentro de las cuatro perspectivas.

2. Esiablecer las relaciones causa-efecto sobre las que se ha construido la estrategia.

3. Si los recursos actuales no son los adecuados para cumplir el objetivo propuesto, ampliar la perspectiva hacia la consecución de nuevas propuestas para satisfacer al clience (logro de la estrategia).

4. Definir, de manera clara, las actividades incernas que se deben cumplir para que la esrrategia tenga éxito.

5. Cruzar las accividades definidas con los procesos seleccionados.

6. Rediseñar los procesos según las nuevas estrategias.

7. Trazar un plan e implementar un programa para volver a definir los procesos (reingeniería).

8. Fabricar indicadores de efecto para que informen si el proceso funciona o no.

9. Identificar el objetivo sobre el cual se deben concentrar los esfuerzos teniendo en cuenta

- Diseño notricial de Plantación Estratergica, que recoge las debilidades, oportunidades. fortalezas y amenazas de una organizarion. mesiame uno matriz estruciurada donde las fortalezas y its debilidades son fortores de fancionamiention intemo. mientras las oporty. nidades y amenozas estón relacionidas con fattores exsernos. los recursos con que la organización cuenta, y mirar si con este esfuerzo se logra aicanzar el éxito (estrategia).

10. Incluir indicadores de inducción o causa para tener señales del cambio, como resultado de la nueva cultura que se pretende implantar.

11. Vigilar si el logro de los objetivos ínternos induce a:

- Crecimiento y aprendizaje

- Mayor acceso a la información

- Incentivar las nuevas conductas

- Mayor productividad

12. Controlar que los indicadores "causa" se traduzcan en

- Incremento de habilidades para el personal

- Acceso a la tecnología e innovación

- Incremento de oportunidad en la información

- Realineación de los objetivos

- Incentivos individuales

13. Los indicadores deben ser recualificados, cuantificados y adecuados para las nuevas exigencias.

14. Se debe fijar un indicador para los riesgos de competencias estrarégicas derivado de la respuesta a las siguientes preguntas

- ¿Cuáles son las comperencias necesarias?

- ¿Qué es lo que tenemos en la actualidad?

- ¿Cuál es la diferencia y qué importancia tiene?

En últimas, para construir un Cuadro Integral de Mando se debe seguir un sistema de pensamiento lógico que defina las prioridades y los indicadores de gestión que mejor arien. ten la estrategia.

Los indicadores deben clasificarse en inductores y de resultado o efecto. Los inductores son los indicadores de causa, que indican lo que se deberia estar haciendo para crear valor. Los inductores sin los indicadores de efecto crean ambigüedad en la forma de alcanzar los resultados. En el cuadro siguience su puede ver un ejemplo. 


\section{Objetivo estratégico}

\section{Desarrollar las competencias necesarias para apoyar el proceso de ventas}

\begin{tabular}{|l|l|l}
\hline $\begin{array}{l}\text { Indicador del resultado } \\
\text { estratégico (efecto) } \\
\text { Ingreso por empleado o } \\
\begin{array}{l}\text { Ventas por vendedor } \\
\text { (causa) } \\
\text { Tasa de cobertura del trabajo } \\
\text { estratégico }\end{array}\end{array}$ & $\begin{array}{l}\text { Iniciativa estratégica } \\
\text { Volver a diseñar el proceso } \\
\text { de desariollo del personal. } \\
\text { Identificar los trabajos } \\
\text { estratégicos. } \\
\text { Construir perfiles. Evaluar el } \\
\text { personal actual. Identificar } \\
\text { desfase. Construir el plan de } \\
\text { desarrollo del personal }\end{array}$ \\
\hline
\end{tabular}

Tomado de Norron y Kaplan : Cuadro de Mando Integrol Pg.168

Los reportes de información deben contener de manera sintetizada los resultados de las variables relevantes con sus tasas de variación, causas de las desviaciones, aspectos del entorno, posibles acciones correctivas, repercusiones y responsables:

La información no debe concebirse como algo centralizado, sino que debe contar con una adecuada coordinación, en muchos casos se sugiere para esto una estructura piramidal donde cada una de las direcciones dispone de su cuadro integral específico que debe destacar las variables mấs relevantes de $5 u$ gestión, nutridos desde fuenres de información de menor responsabilidad, retroalimentada por información horizontal. A la dirección general sólo deben llegar informes verificados en cada uno de los escalones por los que pasa, dando garantía de fiabilidad y permitiendo antelación a las acciones correctivas.

VENTAJAS EN LA IMPLEMENTACIÓN DEL BALANCE SCORECARD

Apunta hacia lograr los ideales cstablecidos en la misión corporativa
Contribuye a que exista una verdadera unidad de dirección dentro de la organización

Hace que los directivos acruen en concordancia con la visión corporativa de la empresa.

Genera una estrategia de divulgación de in" formación de acuerdo a las necesidades de cada nivel administrativo

Crea un ambiente de participación y compromiso entre cada uno de los integrantes de las diversas áreas y entre las áreas mismas

Permite a los participantes conocer los efectos de su desempeño

Fija periodos de ciempo para alcanzar los objetivos propuestos

\section{ALGUNAS DIFICULTADES PARA LA IMPLEMENTACIÓN DEL BALANCED SCORECARD}

El Cuadro Integral de Mando se planrea como una herramienta para la planeación de la organización; su principal reto consiste en 
darle una aplicación adecuada considerándolo como un elemento de proyección y no como un elemento de control histórico.

- Utilizarlo solamente como control interno de la empresa, ya que esto desvirtúa su propósito de contemplar todas las acrividades del entorno que afecten la organización.

- Implantarlo sin el compromiso de la alta gerencia.

- No establecer previamente las políticas de incencivos.

- No utilizarlo para corregir las desviaciones de los objetivos en el momento oportuno.

Utilizarlo sin tener en cuenta la relación causa efecto dentro de las perspectivas.

\section{CONCUUSIONES}

$\Delta$ El artículo conceptualiza una teoria de Planeación Administrativa que integra la gestión de las diferentes áreas funcionales en ргосura del alcance de la misión y visión de la empresa.

A Una organización no puede confiar al azar su desarrollo; requiere de la aplicación sistemácica de técnicas que permitan dimensionar los logras que es dable alcanzar, y el proceso que debe seguirse para llevarlos a feliz término.

$\Delta$ El modelo integra diferentes puncos de vista de una organización para orientarlos hacia una meta específica.

$\Delta$ Un inconveniente que se encuentra al realizar este tipo de trabajos es lograr que se revalúen los diferentes paradigmas que se tienen sobre la administración de las empresas, empezando desde la alta gerencia.

- La integración de las perspectivas planteadas por el B.S.C., nos permite reafirmar que la gestión empresarial no se puede juzgar únicamente desde el punto de vista financiero.

- Si bien el Balanced Scorecard es una herramienca de gestión estratégica, presenta inconvenientes en su aplicación sobre todo por querer limitar la operación de la empresa a tan sólo cuatro perspectivas.

- En la práctica un Cuadro Integral de Mando tiene que escar de acuerdo a las condiciones estructurales de la empresa especifica que se pretende concrolar, teniendo presente, eso si, una unidad de propósito, por cuanto rodo tiende a conseguir una estrategia acorde con las necesidades.

\section{BIBLIOGRAFÍA}

AMAT Oriol: EVA "Valor Economico Agregado". Norma, (1999).

BAIN David: Produccividad "La solución a los problemas de Ja Empresa", McGraw-Hill.

CANADA thon R, Sullivan William, Whice Jhon: Análisis de la Inversión de Capical para Ingeniería y Administración. Prentice-Hall Hispanoamericana. S.A. (1996).

KAPLAN Robert, Norton David: El Cuadro de Mando Integral, KPMG Latinoamericana, (1957).

López Viñegla Alfonso: El Cuadro de Mando y los Sistemas de Información para la Gestión Enpresarial. Posibilidad de Tratamiento Hipermedia. AECA "Asociación Española de Contabilidady Adminustración de Empresas (1998).

MARTínez R. Ricardo: Indicadores Financieros bajo la óptica de Balanced Scorecard. Seminario ACEF (1999).

OCLIASTRI Enrique: Manual de Planeación Estratégica, Tercer Mundo Edicores (1991)

SERNA Cómez. Humberto: Planeación y Gestión Estratégica. RAM Editores (1996). 